\title{
Update: looking beyond the 100,000 Genome Project
}

\author{
"Though the idea of incorporating genomic data in health \\ risk assessments has been raised, the benefits of universal-genome \\ sequencing are far from clear in healthy patients, and even \\ for some diseases."
}

First draft submitted: 28 November 2016; Accepted for publication: 20 December 2016; Published online: 19 January 2017

Keywords: big data • clinical implementation $\bullet$ communication $\bullet$ patient engagement - provider training $\bullet$ sequencing

Announced in 2012, the national UK 100,000 Genome Project created excitement and hope that by sequencing thousands of Britons, more clues about cancer and rare diseases would be identified, leading to new diagnostics and therapies. In addition, participants would be informed of clinically relevant findings that may provide a diagnosis or impact clinical management. As the end of the project nears in 2017, now is a good time to reflect on the progress, but more importantly, look ahead toward the next steps of achieving the penultimate goal of sequencing the genome of every patient in the National Health Service (NHS; 50 million).

\section{Current status}

As of 7 November, almost 15,000 (14,946) genomes have been sequenced, up from 11,221 reported on 4 July 2016. Most of the initial 5000 sequences were from samples collected through pilot projects, as the 13 clinical recruitment centers were still being established and had not begun to recruit patients until March 2015. The project will rapidly accelerate its recruitment effort to achieve its goal of sequencing 85,000 samples over the next year. Recruitment challenges are common throughout clinical trials research [1], and are understandably more complicated in such a large and complex endeavor. As with any project of this scale, development of the necessary infrastructure for recruitment, patient and provider education, sample col- lection, sequencing, analysis and data storage required substantial time and effort, delayed enrollment for a lengthy period after Genomics England was selected to lead the project. In January 2016, the first sick children received diagnoses from the project, achieving one of the project's goals of providing a diagnosis.

\section{The next phase}

Looking ahead, what should the next goal be after the initial phase (sequencing 100,000 samples from 70,000 patients) is completed? If the ultimate goal is clinical implementation of whole genome sequencing or whole exome sequencing for all NHS patients, careful examination is needed to determine if that strategy is still a medically appropriate goal (particularly in light of healthcare costs) and if an adequate NHS-wide infrastructure (training, clinical support and patient resources) can be developed to support this goal. If sequencing is to be provided to all NHS patients, this will obviously include healthy and affected patients and patients of all ages and races/ethnicities. Though the idea of incorporating genomic data in health risk assessments has been raised [2], the benefits of universal-genome sequencing are far from clear in healthy patients [3,4], and even for some diseases [5]. An opportunity exists to examine this population more closely in the 100,000 Genomes Project since approximately 34,000 participants will be unaffected

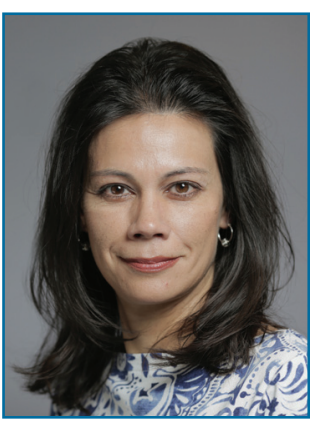

Susanne B Haga

Center for Applied Genomics \& Precision Medicine, Duke University School of Medicine, 304 Research Drive, Box 90141, Durham, NC 27708, USA

Tel.: +19196840325

Fax: +1 9196136448

susanne.haga@duke.edu 
(parents of affected children). Such research on healthy participants has also begun in the US with projects such as HealthSeq and BabySeq supported by the US NIH. If the benefit of genome sequencing of healthy individuals is not demonstrated, an alternative route to consider may be implementation of gene sequencing panels for specific disease risks (if indicated clinically) or for traits where interpretation is unaffected by clinical history or manifestation [6]. For the latter type, one such area may be pharmacogenetics, whereby the interpretation of the variant (regarding its functional significance) will not be impacted by patient's health history and the case can be made for pre-emptive testing [7]. However, the costs of gene testing panels compared with whole-genome sequencing/whole-exome sequencing must be considered.

\section{"...the next phase of the project could expand the recruitment centers to include general practice clinics to enroll the next 100,000 patients, with a key focus on the training and required clinical support."}

Several additional challenges with sequencing healthy individuals need to be considered. The interpretation of genetic variants in healthy individuals may be more difficult, particularly in the absence of family history, which may limit the amount of information that can be derived from sequencing. Furthermore, if genomic data are to be incorporated into heathrisk assessments, more research on a diverse group of healthy patients is needed to provide understanding of rare variants and potentially differential clinical effects. Lastly, and probably most importantly, the interaction of gene and environmental factors requires ongoing study. Several projects have begun to collect a broad swath of data to develop health risk assessments, including genomics and other -omics datasets [8]. The inclusion of environmental exposure data (exposome) $[9,10]$ will be important to inform risk predictions and guide preventive care. Patient-reported outcomes have been broadly incorporated in research [11], often using mobile health applications to collect and transmit data.

Expanding the data generated per participant should also be considered in the next phase of the project. Much progress has been made on our understanding of the microbiome, epigenome and transcriptome data and the impact on health. While the host sequence data remains a central feature of assessing disease risk, the other datasets can provide a better 'snapshot' of the participant's health. However, the transient nature of these other datasets also means that repeated sampling would be needed to determine changes and associations with current and future health.

Enrollment in the 100,000 Genome Project has been limited to patients with cancer and rare diseases. These conditions are typically cared for by health providers who are quite experienced with genetic testing and/or are geneticists. However, clinical implementation of genome sequencing across all practices will present a substantial challenge. General or non-genetic specialty practitioners will require substantial training and clinical support to achieve an adequate level of knowledge and skills to discuss testing and test results with patients and to appropriately apply the results to clinical management. Early assessment of provider attitudes about sequencing in healthy patients suggests that consultation with a genetics expert is desirable [12], which may severely limit clinical integration since genetics experts are limited. Thus, the next phase of the project could expand the recruitment centers to include general practice clinics to enroll the next 100,000 patients, with a key focus on the training and required clinical support.

In my prior editorial [13], I mentioned the importance of effective patient-provider communication for successful implementation of clinical sequencing. While face-to-face is likely the most preferable and effective mode of communication for many patients, the volume of results, the limitations of the information, the limited number of genetic specialists if needed and the recommended follow-up care may require new delivery models. While some groups have developed succinct reporting formats for results from sequencing [14], additional work may be needed to develop patient-friendly reports, such as interactive formats that can easily provide updated information based on current literature and variant interpretation, setting patient preferences about the type of information desired, including links to obtain additional information or explanation, and using audio or video messages of key points.

Since the launch of the 100,000 Genome Project, several other projects of the same scale or larger have launched or been completed by academia and/or industry [15-17] as well as national sequencing projects. Most notably, the US Precision Medicine Initiative was announced in 2015 [18], and expected to begin enrollment of 1 million participants in early 2017. With multiple national programs, perhaps it is worthwhile to consider an international consortium of sequence data and phenotype data to enable greater and more diverse data. Data sharing has not only become acceptable, it is almost an expectation of large studies. For example, the Global Alliance for Genomics and Health is working to establish a framework for data sharing among various stakeholders [19]. Along these lines, a memoran- 
dum of understanding was established in 2015 between Genome British Columbia and Genomics England to share data and work together to develop tools to be applied to clinical interventions and diagnostics. There are some countries for which a national sequencing initiative is not a top priority, yet they have similar disease burdens as the US, UK and Japan, and could benefit from easier access to these data.

\section{Conclusion}

In conclusion, the scope of the 100,000 Genomes Project was amazing at its launch, but the pace of research, technology development, provider needs and patient engagement have continued to accelerate. In 2006, 6 years prior to the establishment of the 100,000 Genomes Project, the UK Biobank was launched to much fanfare. The movement from national and large-scale biobanks entailing collection and storage of thousands of biospecimens to large-scale personal

\section{References}

1 Caldwell PH, Hamilton S, Tan A, Craig JC. Strategies for increasing recruitment to randomised controlled trials: systematic review. PLoS Med. 7(11), e1000368 (2010).

2 Sanderson SC. Genome sequencing for healthy individuals. Trends Genet. 29(10), 556-558 (2013).

3 Lu C. Economic evaluation of whole-genome sequencing in healthy individuals: what can we learn from CEAs of wholebody CT screening? Genet. Med. 18(1), 103-104 (2016).

4 Diamandis EP, Li M. The side effects of translational omics: overtesting, overdiagnosis, overtreatment. Clin. Chem. Lab. Med. 54(3), 389-396 (2016).

5 West HJ. No solid evidence, only hollow argument for universal tumor sequencing: show me the data. JAMA Oncol. 2(6), 717-718 (2016).

6 Judkins T, Leclair B, Bowles K et al. Development and analytical validation of a 25 -gene next generation sequencing panel that includes the BRCA1 and BRCA2 genes to assess hereditary cancer risk. BMC Cancer 15, 215 (2015).

7 Van Driest SL, Shi Y, Bowton EA et al. Clinically actionable genotypes among 10,000 patients with preemptive pharmacogenomic testing. Clin. Pharmacol. Ther. 95(4), 423-431 (2014).

8 Hood L, Lovejoy JC, Price ND. Integrating big data and actionable health coaching to optimize wellness. BMC Med. 13, 4 (2015).

9 Dennis KK, Auerbach SS, Balshaw DM et al. The importance of the biological impact of exposure to the concept of the exposome. Environ. Health Perspect. 124(10), 1504-1510 (2016).

10 Wild CP, Scalbert A, Herceg Z. Measuring the exposome: a powerful basis for evaluating environmental exposures and cancer risk. Environ. Mol. Mutagen. 54(7), 480-499 (2013). genome sequencing projects represents an organic fusion between population-based sciences, genomic medicine and bio- and health informatics. The wall between research and clinical care has become more of a semipermeable cell membrane, enabling exchange of many different types of data to foster learning and evidence generation [20]. The next iteration will continue to increase in scale, with numerous datasets likely per participant, but priorities should shift from discovery to broad clinical implementation.

\section{Financial \& competing interests disclosure}

SB Haga is partly supported by the NIH (R01GM081416). The author has no other relevant affiliations or financial involvement with any organization or entity with a financial interest in or financial conflict with the subject matter or materials discussed in the manuscript apart from those disclosed.

No writing assistance was utilized in the production of this manuscript.
11 Schwartzberg L. Electronic patient-reported outcomes: the time is ripe for integration into patient care and clinical research. Am. Soc. Clin. Oncol. Educ. Book 35, e89-e96 (2016).

12 Cutting E, Banchero M, Beitelshees AL et al. User-centered design of multi-gene sequencing panel reports for clinicians. J. Biomed. Inform. 63, 1-10 (2016).

13 Haga SB. 100k Genome Project: sequencing and much more. Per. Med. 10(8), 761-764 (2013).

14 Vassy JL, McLaughlin HM, MacRae CA et al. A one-page summary report of genome sequencing for the healthy adult. Public Health Genomics. 18(2), 123-129 (2015).

15 Hawkes N. Genetic project calls for 100,000 volunteers from Pakistani and Bangladeshi communities in east London. BMJ 350, h1415 (2015).

16 Carey DJ, Fetterolf SN, Davis FD et al. The Geisinger MyCode community health initiative: an electronic health record-linked biobank for precision medicine research. Genet. Med. 18(9), 906-913 (2016).

17 Kvale MN, Hesselson S, Hoffmann TJ et al. Genotyping informatics and quality control for 100,000 subjects in the genetic epidemiology research on adult health and aging (GERA) cohort. Genetics 200 (4), 1051-1060 (2015).

18 Collins FS, Varmus H. A new initiative on precision medicine. N. Engl. J. Med. 372(9), 793-795 (2015).

19 Lawler M, Siu LL, Rehm HL et al. All the world's a stage: facilitating discovery science and improved cancer care through the global alliance for genomics and health. Cancer Discov. 5(11), 1133-1136 (2015).

20 Chambers DA, Feero WG, Khoury MJ. Convergence of implementation science, precision medicine, and the learning health care system: a new model for biomedical research. JAMA 315(18), 1941-1942 (2016). 